\title{
ARTIGOS
}

\section{ANÁLISIS DE GÉNERO DE LAS ESTRATEGIAS DE CUIDADO INFANTIL EN URUGUAY}

KARINA BATTHYÁNY • NATALIA GENTA • SOL SCAVINO

\section{RESUMEN}

Este artículo tiene como objetivo aproximarse a las estrategias de cuidado infantil desarrolladas por las familias relacionadas a la utilización de cuidados institucionales y/o familiares -la distribución entre varones y mujeres en ese cuidado- así como a las combinaciones entre estos elementos. Los resultados evidencian que las estrategias están basadas en el cuidado familiar y femenino, lo que tiene consecuencias en las altas proporciones de mujeres que abandonan el mercado de trabajo. Dicho abandono tiene consecuencias en términos de la autonomía económica presente y futura de las mujeres, quienes de esta forma asumen individualmente los costos del cuidado.

\section{GÉNERO • CUIDADO DE NIÑOS • ESTRATEGIAS • REPRESENTACIONES SOCIALES}

\section{GENDER ANALYSIS OF CHILD CARE STRATEGIES IN URUGUAY}

\section{ABSTRACT}

This article aims to discuss child care strategies developed by families, related to institutional and/or family care - the sharing of responsibilities between men and women in this care - as well as the combinations of such elements. The results show that the strategies are based on the family and women's care-giving, leading thus to high proportions of women leaving the job market. Such leaving produces consequences with regard to women's present and future economic autonomy. Women, as a result, individually assume the burden of care. 


\section{ANALYSE DE GENRE DES STRATÉGIES DE GARDE D'ENFANTS EN URUGUAY \\ RÉSUMÉ}

Cet article présente les strategies de garde d'enfants des familles, le recours aux equipements institutionnels ou aux soins familiaux - la distribution des et des femmes dans ce soin -, ainsi que les combinaisons entre ces éléments. Les résultats mettent en évidence que les stratégies sont basées sur le soin familial et féminin, en conséquence une proportion élevée de femmes abandonne le marché de travail. Cet abandon a um impact sur l'autonomie économique présente et future des femmes, qui assument individuellement les coûts du soin.

GENDER • GARDE D’ENFANT • STRATÉGIE • REPRESENTATIONS SOCIALES

\section{ANÁLISE DE GÊNERO DAS ESTRATÉGIAS DE CUIDADO INFANTIL NO URUGUAI}

RESUMO

Este artigo tem o objetivo de apresentar as estratégias de cuidado infantil desenvolvidas pelas famílias, relacionadas com o uso de cuidados institucionais e/ou familiares - a distribuição de homens e mulheres nesse cuidado -, bem como as combinações entre tais elementos. Os resultados evidenciam que as estratégias estão baseadas no cuidado familiar e feminino, o que produz como consequência as altas proporções de mulheres que abandonam o mercado de trabalho. Tal abandono produz consequências no tocante à autonomia econômica presente $e$ futura das mulheres, que assim assumem individualmente os custos do cuidado. 
NO DE LOS CAMBIOS SOCIALES MÁS IMPORTANTES EN LAS ÚLTIMAS DÉCADAS ES la masiva y persistente incorporación de las mujeres al mercado de trabajo remunerado. Sin embargo, permanece sin modificación su participación y responsabilidad en las actividades sin remuneración del ámbito doméstico, entre ellas los cuidados, los cuales continúan estando mayoritariamente a cargo de las mujeres en las redes familiares.

Este artículo tiene como objetivo aproximarse a las estrategias de cuidado infantil de niños/as pequeños/as desarrolladas por las familias, relacionadas a la utilización de cuidados institucionales y/o familiares, verificando la distribución entre varones y mujeres en ese cuidado, así como las combinaciones entre estos elementos. El trabajo de cuidados realizado en el ámbito del hogar presenta particularidades debido a los aspectos materiales y afectivos que lo componen. Requiere reconocer las necesidades de los otros y ejecutar tareas tanto materiales como aquellas que apuntan al desarrollo del vínculo afectivo, para satisfacer dichas necesidades. Siguiendo a Tronto (2010) existen al menos tres elementos requeridos para brindar cuidados: reconocer las necesidades de cuidado, responsabilizarse por las tareas y ejecutarlas. Las mujeres son asociadas con las habilidades naturales para reconocer las necesidades de los otros/ as y para la ejecución de las tareas, al mismo tiempo que se las considera responsables del cuidado.

La división sexual del trabajo, plenamente vigente, está estrechamente vinculada con un modelo de familia que se naturalizó 
como el único posible. Este modelo se caracteriza por estar conformado por una pareja heterosexual con hijos de ambos en la que el varón es el proveedor de ingresos y la mujer es la encargada del trabajo doméstico y de cuidados (BECK; BECK-GERNSHEIM, 2012). El rol de las mujeres en este modelo de familia es naturalizado y el cuidado a dependientes es colocado como una tarea "hecha por amor", aspecto relacionado estrechamente con una determinada concepción naturalizada sobre la maternidad. En este sentido el amor-materno se ha configurado como el sustento ideológico del cuidado familiar-femenino por excelencia y de un modelo único ideal de familia (BOCK; DUDEN, 1985).

Desde hace algunas décadas y hasta la actualidad, la incorporación de las mujeres en el mundo laboral abre un proceso de re-significación de la maternidad que incorpora el conflicto permanente con la realización de las actividades del mundo productivo ya que las continúa colocando como responsables naturales del cuidado, al mismo tiempo que crece su participación laboral y sigue sin modificarse el involucramiento masculino en los cuidados (CARRASCO; BORDERÍAS; TORNS, 2011).

La inserción de las mujeres al mundo laboral requiere que el trabajo doméstico y de cuidados naturalizado como propio del rol femenino deba ser racionalizado y derivado parte del mismo a otros agentes, como el mercado, el Estado, la comunidad y/o a los varones. Esta derivación y distribución de los recursos de tiempo y dinero termina configurando una determinada estrategia de cuidados que se vuelve especialmente relevante de analizar en la medida en que las mujeres acceden al mercado laboral.

En este trabajo, las estrategias de cuidado serán consideradas como elecciones de las personas que integran los hogares, pero productos de la combinación de factores de naturaleza estructural (división sexual del trabajo en los hogares, posición de clase, segregación por sexo del mercado laboral, disponibilidad de acceso material a servicios de cuidado en el mercado y provistos por el Estado, entre otras) con factores culturales ( mandatos de género, actitudes, valoraciones y normas sociales que determinan el ideal de cuidado y las personas ideales para ejercerlo). Estos últimos son particularmente relevantes, ya que las modalidades de cuidado ideal y/o deseables pueden actuar como resistencias socioculturales a las acciones de política pública que pretendan desfamiliarizar los cuidados, como lo son por ejemplo las instituciones de cuidado para niños/as pequeños/as.

\section{ESTRATEGIAS DE CUIDADO INFANTIL}

En la teoría sociológica contemporánea podemos encontrar desarrollos diversos de la noción de estrategias relacionadas a la concepción sobre la racionalidad de los agentes sociales y al vínculo entre las posiciones 
individuales y la estructura social. Estos desarrollos provienen al menos de dos abordajes; del individualismo metodológico, centrados en la racionalidad instrumental (ELSTER, 1988) y por otro lado de las teorías de articulación agencia y estructura de Giddens y Bourdieu, quienes retoman y relocalizan la noción de racionalidad práctica para la teoría sociológica.

En este artículo, las estrategias de cuidado se conciben como el resultado de una serie de acciones que combinan constricciones estructurales y culturales con elementos voluntarios o de agencia individual. Dichas estrategias configuran en el plano individual una determinada distribución del cuidado entre los distintos agentes proveedores.

Wallace (2002) expone la relevancia del análisis de las estrategias destacando que tienen un resurgir teórico vinculado a los procesos de reflexividad y concientización propios de las sociedades actuales, que hace que la organización de los recursos de tiempo y dinero que llevan a cabo los hogares tengan un gran valor analítico.

La idea de estrategia, criticada en sus primeras versiones por obedecer pura y exclusivamente a una teoría económica-instrumentalista del comportamiento social, actualmente se concibe como producto de la intersección de elementos estructurales (materiales), culturales (normativos, valorativos) y de las motivaciones individuales (WALLACE, 2002).

Por su parte, Tobío (2002) estudia las estrategias para la superación de la contradicción familia-empleo en el caso español, observando que las mismas aparecen como reacción a un momento de cambio social en el que las mujeres se incorporan al mercado de trabajo de manera masiva a la vez que cambian las estructuras familiares y aumentan las demandas de cuidado para la población dependiente. A su vez, no existen instituciones preparadas o pautas establecidas sobre cómo hacer frente a dichos desajustes. Esto se traduce en un conflicto personal entre distintos roles asumidos por las mujeres: de madre y de trabajadora remunerada. En su trabajo, presenta una categorización múltiple de estrategias llevadas a cabo por las mujeres trabajadoras con niños/as pequeños/as. Las denominadas "estrategias principales” son suficientes por sí mismas para responder a demandas domésticas. Consisten sobre todo en la participación clave de la abuela materna y en una casi nula participación de los varones. Las “estrategias complementarias” se definen así porque no son suficientes para responder a la totalidad de la demanda de cuidado, pero muchas veces son necesarias para el desarrollo de la estrategia principal. Ejemplos de estas estrategias son la reducción de distancias a la casa de la abuela materna, a las empresas donde se trabaja y la simplificación del trabajo doméstico a través de contrataciones de empleadas domésticas que permitan reducir una parte de la carga. 
Las estrategias denominadas "indirectas" son de tipo extremo y son negativas porque no son deseadas (por ejemplo, faltar al trabajo si el hijo/a está enfermo). En este caso la compatibilidad entre trabajo y cuidados se asegura eliminando o reduciendo parte del problema; disminuyendo su participación en el mercado de empleo, reduciendo la cantidad de hijos o directamente no teniéndolos. Este artículo, como ya fue mencionado, se aproximará al análisis de las estrategias de cuidado de niños/as pequeños/as implementadas en Uruguay a partir de indicadores cuantitativos disponibles en el país.

\section{LA ORGANIZACIÓN SOCIAL DEL CUIDADO EN URUGUAY}

En la definición de las estrategias de cuidado de las familias intervienen factores culturales. Nos referimos a aquellos mandatos de género que definen las expectativas y obligaciones asociadas a madres y padres y el nivel de familismo, entendido como la responsabilidad que se le asigna a las familias en el cuidado, todos elementos que orientan las prácticas de cuidado y que contribuyen de este modo con la definición de la estrategia en el hogar.

Junto a dichas valoraciones culturales, las estrategias de cuidado están influidas por el contexto de políticas de cuidado que se experimenten. De esta forma las elecciones que se tomen estarán condicionadas por las políticas públicas de cuidado a las que potencialmente se pueda acceder. Estas políticas son parte de las condiciones estructurales de la organización social del cuidado en las que se desarrollan las estrategias de cuidado.

Las políticas de cuidado pueden clasificarse en cómo se reasignan los recursos (tiempo, ingresos y servicios). A esta clasificación puede sumarse un análisis que distinga entre ellas los elementos maternalistas (aquellos que reproducen la división sexual del trabajo y fomentan un cuidado familiar y materno no remunerado), y los que promueven un cuidado corresponsable (en el cual se prevé participación estatal y del mercado que contribuye a desfamiliarizar el cuidado y estimula la participación activa de los varones en el cuidado) (BLOFIELD; MARTINEZ, 2014).

Las políticas de reasignación de tiempo son acciones para que los tiempos de trabajo y de cuidado familiar se articulen de manera equilibrada, asegurando los ingresos para suplir el trabajo remunerado durante determinados períodos de tiempo. La flexibilización horaria, las licencias de maternidad, paternidad, parentales y reducciones horarias para cuidados, son algunos ejemplos de políticas de esta clase. En este tipo de políticas, si bien el Estado asume parte de los costos del cuidado, también se favorece que el cuidado se mantenga en el ámbito familiar. Estas medidas, frecuentemente, no son de gran ayuda a la hora 
de redistribuir la inequidad en la división sexual del trabajo, dado que suelen ser utilizadas por mujeres cuando las mismas no desarrollan mecanismos explícitos para que lo hagan los varones.

Las políticas de reasignación de ingresos ofrecen dinero para solventar el cuidado infantil independientemente de que sea realizado por un familiar o no, como es el caso de las transferencias monetarias. En muchos casos, los escasos montos de transferencias fomentan el cuidado familiar y a cargo de mujeres. Por su parte, los servicios de cuidado infantil presentan las ventajas de poder desfamiliarizar el cuidado, porque permiten delegar parte del cuidado desde las familias hacia instituciones (FERNANDEZ; TOBÍO, 2006).

En la mayoría de los países de la OCDE y países latinoamericanos la oferta de servicios de cuidados infantiles a partir de los 4 años de edad es amplia. Sin embargo, existe un vacío generalizado de la cobertura en los niños entre 0 y 3 años. Países como Dinamarca, Suecia, Islandia y Bélgica tienen tasas superiores a 30\% de cobertura, mientras que Austria, Alemania Occidental, Grecia, Irlanda, el Reino Unido, España tienen una cobertura menor al 5\% (FERNANDEZ; TOBÍO, 2006).

En Uruguay, la provisión del cuidado está basada principalmente en el aporte de las mujeres y las familias, con escasa participación del Estado ${ }^{1}$, a través de programas focalizados que atienden parcialmente

En la actualidad, el país se propone el aumento de la oferta de servicios y/o prestaciones de cuidado a partir de la instalación del Sistema Nacional de Cuidados SNC. EI SNC tiene entre sus cometidos promover la corresponsabilidad de los cuidados entre varones y mujeres y entre Estado, mercado, familias y comunidad. Por tanto tiene la pretensión de generar un cambio en la actual organización social del cuidado. partir de los 4 años aunque los servicios públicos

de cuidado no abarcan la jornada de trabajo completa (MIDES, 2014).

Se encuentra un resumen sobre las Licencias en Uruguay y su impacto de género en Batthyány, Genta y Perrotta (2015) "Avanzando en la corresponsabilidad en los cuidados: el caso de las licencias parentales en Uruguay". Serie Asuntos de Género Nro. 128. Cepal, Santiago a la población vulnerable, y con una oferta del mercado que solo cubre a la población que puede pagar los costos que implican los servicios ofrecidos.

Así, la cobertura pública de centros de cuidado infantiles es muy escasa. Tan solo el 33,2\% de los niños/as pequeños/as en Uruguay (0 a 3 años) asisten a algún centro de cuidado infantil (MIDES, 2014). ${ }^{2}$

La escasa oferta de servicios públicos de cuidados acentúa algunas desigualdades socioeconómicas, ya que mientras que las mujeres de clases altas pueden pagar los costos de un centro infantil o una persona contratada en el hogar, las provenientes de clases bajas acceden a programas específicos de cuidados y las mujeres de clases medias trabajadoras son las más perjudicadas por el conflicto trabajo-cuidados.

A su vez, las leyes que permiten articular el trabajo remunerado con el cuidado son escasas y están dirigidas fundamentalmente a las mujeres. ${ }^{3}$ Existen tres tipos de licencias: las licencias por maternidad, paternidad y parentales. La licencia maternal tiene una duración de 14 semanas en empleos del sector privado y 13 en el sector público. Las licencias paternales, de tímido desarrollo en el país, tienen una duración de 10 días en el sector privado y público.

Desde 2013 Uruguay dispone de licencias parentales para trabajadores independientes y del sector privado. Consisten en una reducción de medio horario para cuidados y pueden utilizarse desde que termina la licencia maternal y hasta los seis meses del recién 
nacido. La licencia puede ser utilizada en forma alternada por madre y/o padre. Los datos disponibles muestran que son las mujeres las que mayoritariamente utilizan las licencias parentales, lo cual es esperable dadas las representaciones sociales respecto al cuidado de los niños y niñas menores de un año, que asocian a las mujeres como principales cuidadoras. La fuerte promoción pública de la lactancia materna exclusiva hasta los seis meses de vida del recién nacido también es un factor a considerar en esta mayor utilización.

Las licencias parentales en Uruguay presentan al menos dos limitaciones como mecanismo de fomento a la corresponsabilidad. Primero, en un marco de representaciones familistas en el cuidado infantil y de mandatos de género muy marcados, que asignan a las mujeres su responsabilidad, la transferibilidad de las licencias y la ausencia de días de uso exclusivo para los padres varones, es un obstáculo para la utilización de la licencia de parte de los varones. Segundo, la superposición del periodo de la licencia con el de promoción de lactancia materna exclusiva desestimula su uso por parte de los varones. Ambos factores son claras limitaciones para que las licencias sean un mecanismo efectivo para generar modificaciones en la división sexual del trabajo de cuidado infantil en los hogares uruguayos.

\section{METODOLOGÍA}

Con el objetivo de aproximarse a las estrategias de cuidado infantil de niños/as pequeños/as desarrolladas por las familias, se ha analizado la información de la Encuesta Nacional de Desarrollo Infantil y Salud (en adelante ENDIS) de 2013. La ENDIS fue llevada a cabo por el Instituto Nacional de Estadística, el Instituto de Economía de la Facultad de Ciencias Económicas de la Universidad de la República y el Programa Uruguay Crece Contigo. Se trata de un módulo adosado a la Encuesta Continua de Hogares y el trabajo de campo fue realizado durante el año 2013. Es una encuesta de tipo panel que tuvo como objetivo relevar información sobre salud, nutrición, desarrollo y prácticas de crianza de niños/as de hasta 4 años con el fin de generar insumos para políticas públicas de infancia. Su unidad de análisis fueron los niños/as de 0 hasta 4 años y su cobertura poblacional, los hogares particulares ubicados en localidades urbanas mayores a 5.000 habitantes. La encuesta fue aplicada a los responsables de la crianza de los/as niños/as. ${ }^{4}$

A pesar de que la ENDIS no tuvo como propósito disponer de información sobre las estrategias de cuidado infantil desarrolladas por las familias en Uruguay, se relevaron algunos indicadores que nos permiten aproximarnos a las mismas.

La Encuesta presentó datos de 3077 niños/as de hasta 4 años, que pertenecen a 2665 hogares uruguayos. El 81,3\% de los hogares

\section{4}

Dado que los casos de los/as niños/as menores de 4 años eran insuficientes para el análisis, se sumaron hogares encuestados en la Encuesta Continua de Hogares (URUGUAY, 2012). 
encuestados tiene un/a niño/a por hogar mientras que en el restante 18,7\% residen más de un/a niño/a.

Cada uno de los/as niños/as encuestados/as tiene una persona de referencia, quien es identificada como responsable de la crianza, en su mayoría madres $(96,6 \%)$ mientras que el $1,6 \%$ de los encargados son los padres. Por tanto, en la mayor parte de los casos, este artículo se centrará en el análisis de la situación de las madres de los/as niños/as.

Utilizando los datos disponibles, este artículo busca explorar las estrategias de cuidado infantil: quiénes son los cuidadores principales, qué instituciones participan y de qué manera, qué tipos de hogares externalizan más el cuidado.

\section{¿CUÁLES SON LAS ESTRATEGIAS DESARROLLADAS PARA EL CUIDADO DE NIÑOS/AS PEQUEÑOS/AS?}

Una de las indagaciones principales de la ENDIS fue conocer quiénes se hacen cargo de manera mayoritaria del cuidado infantil. Casi la totalidad de los casos (99\%) manifiesta contar con la madre de los/as niños/as para su cuidado, sin embargo tan solo 6 de cada 10 plantea que cuenta con el padre para la realización de dicha tarea. Aparece en tercer lugar la abuela o abuelo $(38,4 \%)$ como estrategia de sostén de esos cuidados cotidianos. Luego otro pariente (tías, primas), y es recién en el quinto lugar que aparece la opción de contar con una persona remunerada. Con porcentajes más reducidos se mencionan hermanos/as y vecinos/as. Estos datos evidencian, en primer lugar, la importancia de la red familiar para el cuidado de los/as niños/as pequeños/as y en segundo lugar la centralidad de las mujeres en las estrategias de cuidado infantil.

Las mujeres aparecen como las principales cuidadoras, ya sea en su rol de madre, pareja del padre o abuelas. La red familiar, principalmente a través de las abuelas, juega un papel clave en la estrategia de cuidado infantil.

La encuesta también indaga las situaciones no previstas en las estrategias de cuidados cotidianos y se plantea la misma situación: son las madres quienes brindan cuidados en primer lugar, aunque es más alto el porcentaje de hogares que acuden a los/as abuelos/as. Por tanto, en casos que se requieren estrategias de cuidado distintas a la cotidiana, es más importante el rol que adquieren los abuelos, probablemente las abuelas.

Estas situaciones de "emergencia" del cuidado dan cuenta de con quienes se cuenta para el cuidado cuando la estrategia principal se ve alterada o falla. La Encuesta indaga por dos situaciones emergentes, ante una enfermedad o cuando no se dictan clases en el centro educativo o de cuidados al que asisten los/as niños/as.

A su vez, la Encuesta de Cuidados No Remunerados en Salud del año 2013 realizada por el Grupo Sociología de Género (BATTHYÁNY; 
GENTA; PERROTTA, 2014) arroja el dato de que efectivamente son las mujeres entre 46 a 65 años (muchas de ellas abuelas) quienes se encargan mayoritariamente de cuidar a niños y adultos ante situaciones emergentes de enfermedad que desbordan la estrategia cotidiana de cuidados (BATTHYÁNY; GENTA; PERROTTA, 2014).

\section{CUADRO 1}

PERSONAS QUE SE ENCARGAN DEL CUIDADO DE LOS/AS NIÑOS/AS EN EL HOGAR Y PERSONAS CON LAS QUE SE CUENTA PARA EL CUIDADO ANTE UNA SITUACIÓN DE ENFERMEDAD O SI NO SE DICTAN CLASES EN EL CENTRO AL QUE ASISTEN. TOTAL PAÍS, 2013

\begin{tabular}{|l|c|c|}
\hline & $\begin{array}{c}\text { PERSONAS QUE SE ENCARGAN } \\
\text { DEL CUIDADO DE LOS/AS NIÑOS/ } \\
\text { AS EN EL HOGAR (\%) }\end{array}$ & $\begin{array}{c}\text { PERSONA CON LA QUE CUENTA } \\
\text { PARA EL CUIDADO ANTE } \\
\text { SITUACIÓN DE ENFERMEDAD O } \\
\text { SI NO SE DICTAN CLASES (\%) }\end{array}$ \\
\hline Madre & 99,0 & 92,3 \\
\hline Padre & 60,0 & 55,5 \\
\hline Abuelo/a & 38,4 & 47,1 \\
\hline Otro pariente & 12,5 & 14,8 \\
\hline Persona remunerada & 11,1 & 9,0 \\
\hline Hermano/a mayor de 10 años & 8,4 & 6,5 \\
\hline Vecinos u otro no pariente & 2,0 & 2,6 \\
\hline Hermano/a menor de 10 años & 1,8 & 1,4 \\
\hline
\end{tabular}

Fuente: Elaboración propia en base a ENDIS, INE-IECON-UCC.

Cuando se releva particularmente qué fue lo que ocurrió la última vez que se enfermó, se aprecia que en la mayoría de los casos la que se quedó al cuidado fue la madre, con los costos que tiene la ausencia en el trabajo remunerado para muchas de estas mujeres. Tan solo en el 23,9\% de los casos se quedaron los padres. En un 12,4\% acudió una abuela y solo en el 1,7\% fueron abuelos varones. Las trayectorias de mujeres y varones en el mercado laboral, sus posibilidades de ascenso, de capacitación, están determinadas por estas situaciones en las cuales son las mujeres las que mayoritariamente asumen el papel de cuidadoras, ausentándose o modificando su situación en el empleo para cubrir estas necesidades, evidenciando la división sexual del trabajo y condicionando la autonomía económica de las mujeres. 
CUADRO 2

PERSONA QUE LO CUIDÓ LA ÚLTIMA VEZ QUE SE ENFERMÓ. TOTAL PAÍS, 2013

\begin{tabular}{|l|c|}
\hline & $\%$ \\
\hline Padre & 23,9 \\
\hline Madre & 55,8 \\
\hline Abuela & 12,4 \\
\hline Persona remunerada & 2,1 \\
\hline Abuelo & 1,7 \\
\hline Hermana & 1,7 \\
\hline Hermano & 0,4 \\
\hline Otros/as & 2,2 \\
\hline Total & 100 \\
\hline
\end{tabular}

Fuente: Elaboración propia en base a ENDIS, INE-IECON-UCC.

Nota: Respuesta múltiple, el porcentaje es sobre el total de las respuestas.

La mayor presencia femenina en los cuidados queda evidenciada también por los resultados de la Encuesta de Uso del Tiempo en Uruguay (2013), que muestran que mientras el 67,6\% de los varones que conviven con niños/as menores de 3 años participa de tareas de cuidado dedicándoles un promedio semanal de 13 horas, las mujeres lo hacen en el 90,1\% de los casos dedicándoles 22 horas semanales en promedio, casi el doble de las proporcionadas por los varones (BATTHYÁNY, 2015). Estos datos muestran el menor involucramiento masculino en las tareas de cuidado infantil.

Como hemos visto hasta ahora, las estrategias de cuidado infantil son esencialmente familiares y femeninas. La participación del Estado o del mercado por medio de cuidadoras/es remuneradas/os aparece tímidamente, siendo tan sólo 1 de cada 10 casos, con una pequeña variación entre la estrategia cotidiana de cuidado y las situaciones emergentes.

Las estrategias de cuidado familiar y femenino coinciden con los ideales de cuidado presentes en la sociedad uruguaya. La Encuesta de Representaciones sociales del cuidado del año 2011 (FCS, UDELAR) aporta información sobre las situaciones de cuidado infantil deseables para la población uruguaya. Dentro de los distintos tipos de cuidados deseables para los niños/as de 0 a 2 años (población con menor oferta de centros de cuidados por parte del Estado y el mercado), se encontró que la modalidad de cuidado domiciliaria es la más deseada por la población (75\%). A su vez, dos tercios de la población (65\%) se inclinan por que el cuidado sea realizado solo por los miembros de la pareja o familiares cercanos (BATTHYÁNY; GENTA; PERROTTA, 2013). Sin embargo, dentro del cuidado domiciliario y familiar se presentan diferentes expectativas y obligaciones para mujeres y varones. Se manifiesta que la situación ideal de cuidado de los/as niños/as de hasta 2 años durante la jornada laboral es aquella provista por las mujeres de la familia, particularmente las madres (BATTHYÁNY; GENTA; PERROTA, 2013). 
El acceso a los servicios de cuidado provistos por el mercado es uno de los elementos que inciden en la configuración de la estrategia de cuidados, así como en las representaciones sociales. Por tanto, la situación respecto a la pobreza es una variable clave para dar cuenta de las diferentes estrategias y representaciones de cuidado infantil. En los hogares no pobres, es mayor la proporción de quienes cuentan con personas remuneradas para cubrir el cuidado. En el caso de las personas pobres, ellas utilizan en mayor medida el cuidado no remunerado de los hermanos mayores de 10 años (probablemente hermanas), con las consecuencias que esto tiene para el tiempo disponible de estos niños/as para estudiar, recrearse y realizar otras actividades.

En cuanto a los ideales de cuidado, la Encuesta de Representaciones sociales del cuidado mencionada evidencia que a menor nivel socioeconómico, las representaciones son más familistas (BATTHYÁNY; GENTA; PERROTTA, 2013).

CUADRO 3

PERSONA CON LA QUE CUENTA PARA EL CUIDADO DE LOS/AS NIÑOS/AS CUANDO NO HAY CLASES O ESTÁN ENFERMOS SEGÚN SITUACIÓN DE POBREZA DEL HOGAR. TOTAL PAÍS, 2013

\begin{tabular}{|l|c|c|c|}
\hline & NO POBRE (\%) & POBRE (\%) & TOTAL (\%) \\
\hline Madre & 99,2 & 98,5 & 99,0 \\
\hline Padre & 65,1 & 46,1 & 60,6 \\
\hline Abuelo/a & 38,6 & 37,7 & 38,4 \\
\hline Otro pariente & 11,2 & 16,5 & 12,5 \\
\hline Persona remunerada & 14,3 & $1,8^{*}$ & 11,3 \\
\hline Hermano/a mayor de 10 años & 7,1 & 13,7 & 8,7 \\
\hline Hermano/a menor de 10 años & $1,2^{*}$ & $2,8^{*}$ & $1,6^{*}$ \\
\hline Vecinos u otro no pariente & 1,9 & $1,4^{*}$ & 1,8 \\
\hline Otros/as & 1,2 & 2,8 & 1,6 \\
\hline
\end{tabular}

Fuente: elaboración propia en base a ENDIS, INE-IECON-UCC.

Nota: Respuesta múltiple, el porcentaje es sobre el total de las respuestas.

*Menos de 30 casos ponderados

En definitiva, las estrategias desarrolladas para el cuidado de los/as niños/as tienen como principal referencia a sus madres. Se recurre al apoyo de otros familiares cercanos, mayoritariamente mujeres que brindan cuidados no remunerados cuando se presentan situaciones no previsibles en torno al cuidado (enfermedad o ausencia de docentes o cuidadores en una institución). En los hogares no pobres, aparece la contratación de personas en el hogar como parte de la estrategia de cuidado, aunque de forma reducida. 


\section{CENTROS DE CUIDADO INFANTIL COMO ESTRATEGIA DE CUIDADO}

Una de las estrategias utilizadas por los hogares para el cuidado de los/as niños/as pequeños/as es la asistencia a establecimientos de cuidado infantil durante la jornada laboral y en combinación o no con el cuidado familiar. La disponibilidad de servicios de cuidado infantil públicos es uno de los mecanismos que permite la articulación de los ámbitos familiares y laborales, al mismo tiempo que promueve la equidad de género y posibilita contar con servicios de calidad (FERNANDEZ; TOBÍO, 2006).

Sin embargo, tan sólo el 34,5\% de los/as niños/as pequeños/as asisten a un centro infantil, mientras que el 65,5\% restante no lo hace. La reducida asistencia está relacionada con la baja oferta de servicios públicos y gratuitos de cuidado para estas edades, las dificultades económicas en el acceso a las ofertas del sector privado y a las representaciones sociales familistas del cuidado.

En el nivel de las representaciones sociales existe una resistencia a cubrir el cuidado infantil con instituciones para los/as niños/as más pequeños. Apenas un grupo cercano al 25\% optaría por compartir el cuidado entre familiares y centro infantil durante la jornada laboral (BATTHYÁNY; GENTA; PERROTTA, 2013).

La cantidad de personas que manifiesta que en términos ideales compartirían la estrategia de cuidado entre hogares y centros infantiles es levemente inferior al porcentaje de niños/as pequeños/as que efectivamente asiste a dichos centros. Por tanto, existe una pequeña disociación entre la disposición por compartir el cuidado con instituciones (25\%, según la Encuesta de Representaciones Sociales del cuidado antes citada) y la asistencia efectiva de los/as niños/as a centros de cuidado (34,5\%), que no cumpliría las demandas más familistas de la población.

Como se observa en el cuadro 4, en cuanto a la asistencia a los centros se encuentran diferencias según la situación de pobreza en el hogar. La asistencia es más baja entre la población pobre $(26,2 \%)$ que entre los no pobres (37,1\%). Lo mismo ocurre con la continuidad de los/as niños/as en la asistencia a los centros, la cual es más baja entre aquellos que integran hogares pobres $(9,4 \%)$ que entre los que provienen de hogares no pobres (5,3\%).

A pesar de que los servicios de cuidado infantil públicos y gratuitos están destinados a las poblaciones pobres, los niños pertenecientes a estos hogares presentan menor asistencia a los centros, lo que evidencia seguramente representaciones sociales más familistas (BATTHYÁNY, 2015). 
ASISTENCIA A ESTABLECIMIENTO DE CUIDADO INFANTIL POR POBREZA DE LOS HOGARES. TOTAL PAÍS, 2013

\begin{tabular}{|l|c|c|c|}
\hline & NO POBRE (\%) & POBRE (\%) & TOTAL (\%) \\
\hline Asisten & 37,1 & 26,2 & 34,5 \\
\hline No asisten & 62,9 & 73,8 & 65,5 \\
\hline Total & 100 & 100 & 100 \\
\hline
\end{tabular}

Fuente: elaboración propia en base a ENDIS, INE-IECON-UCC, 2013.

La asistencia al centro infantil está influenciada por la participación laboral de las mujeres. Esto queda evidenciado en el dato que muestra que entre las madres que trabajan de forma remunerada, el 37,6\% de los/as niños/as asisten actualmente a algún centro infantil, mientras esto ocurre con el $23,5 \%$ de los/as niños/as cuyas madres no trabajan. El acceso al empleo de las mujeres en los hogares no pobres, incrementa la asistencia de los niños/as a centros de cuidado infantil.

Existe una relación entre la situación de pobreza de los hogares y la participación laboral de las mujeres, ya que aquellas que viven en hogares pobres tienen tasas de empleo inferiores a las no pobres (URUGUAY; MIDES, 2013).

Por tanto, la baja asistencia de los niños en hogares pobres puede ser entendida por el hecho de que en estos hogares prima una división sexual del trabajo tradicional a partir de la cual las madres tienen un menor o nulo acceso al trabajo remunerado y se encuentran disponibles totalmente para el cuidado.

CUADRO 5

ASISTENCIA A CENTRO DE CUIDADO INFANTIL, SEGÚN ACTIVIDAD LABORAL DE LAS MUJERES MADRES. TOTAL PAÍS, 2013

\begin{tabular}{|l|c|c|c|}
\hline & TRABAJA (\%) & NO TRABAJA (\%) & TOTAL (\%) \\
\hline Asisten & 37,6 & 23,5 & 32,0 \\
\hline No asisten & 62,4 & 76,5 & 68,0 \\
\hline Total & 100 & 100 & 100 \\
\hline
\end{tabular}

Fuente: Elaboración propia en base a ENDIS, INE-IECON-UCC.

\section{MOTIVOS DE ASISTENCIA Y DE NO ASISTENCIA A CENTROS DE CUIDADO INFANTIL}

Cuando indagamos entre los/as niños/as que no asisten a los centros infantiles los principales motivos son: la edad temprana para asistir a establecimientos $(35,4 \%)$ seguido de la opción del hogar por el cuidado familiar (34,4\%). En tercer lugar, aparecen los problemas de tipo logístico relacionados a los servicios que se ofrecen, como la lejanía, los problemas de transporte o de incompatibilidad con horarios de trabajo, los que sumados no llegan al 4,9\% de los casos.

Respecto al motivo relacionado a la edad temprana de los niños, es coincidente con lo planteado por un grupo influyente de expertos 
en cuidado infantil, como ha demostrado una investigación que se propuso indagar las claves del buen cuidado infantil en base al saber experto (BATTHYÁNY; GENTA; PERROTTA, 20145). Uno de los hallazgos principales de este estudio, es la identificación de tres enfoques dentro del saber experto a la hora de definir el cuidado de calidad y sus dimensiones centrales, en los que se identifican énfasis diferenciados, así como diversas posturas sobre el ejercicio del derecho a cuidar desde una perspectiva de género y derechos.

A la hora de las recomendaciones sobre el buen cuidado, así como en la definición del mismo, el enfoque denominado "Médico" se centra en los aspectos sanitarios del cuidado: el garantizar la lactancia materna y la prevención de enfermedades. La centralidad que tiene para este enfoque la lactancia materna, y las recomendaciones acerca de la importancia de que los/as niños/as menores de dos años permanezcan cuidados en el hogar, para prevenir enfermedades, lleva a que este enfoque sea más "familista”, es decir, centrado en que los cuidados de calidad son provistos únicamente por la familia, y que sea entonces menos habilitador para la perspectiva de género y derechos, dado que refuerza los roles de género tradicionales, manteniendo a las mujeres como principales cuidadoras (BATTHYÁNY; GENTA; PERROTTA, 2014).

La investigación también evidencia la existencia de otro discurso denominado "Psicológico-educativo", el que habilita otras posibilidades de cuidado de calidad, al destacar que lo que determina el buen cuidado son los vínculos seguros que pueden ser desarrollados con otros adultos/as referentes incluso con cuidadores/as remunerados/as en domicilio o que trabajen en centros infantiles (BATTHYÁNY; GENTA; PERROTTA, 2014).

Por tanto la opción por el cuidado institucional potencialmente puede brindar calidad en el cuidado de los/as niños/as, pero aparece como una opción poco legitima para la población.

Es importante tener presente que el discurso médico goza de importante respeto y legitimidad en la sociedad, incidiendo fuertemente en las prácticas, sobre todo en los aspectos de crianza de la primera infancia, por lo que las consecuencias en las prácticas de cuidado, tanto si es posible llevar adelante sus recomendaciones como si no lo es, pesan fuertemente en la experiencia de las familias y en las mujeres respecto a las decisiones y estrategias de cuidado. Dicha legitimidad queda evidenciada, por ejemplo en la Encuesta de Representaciones sociales del cuidado (BATTHYÁNY; GENTA; PERROTTA, 2014) mediante la cual se muestra que si bien un tercio de la población consulta sobre los aspectos de crianza en primer lugar a sus madres, una cuarta parte de las mujeres consulta en primer lugar a los médicos o personal de la salud, lo que evidencia el lugar de privilegio que tiene este saber en las prácticas y representaciones del cuidado infantil (BATTHYÁNY; GENTA; PERROTTA, 2014). 
De este modo, no es extraño que como primer motivo para no enviar a los niños a centros de cuidado infantil encontremos la edad temprana de los/as niños/as y la prevención de enfermedades, ambos aspectos que pueden asociarse al discurso "Médico" que se centra en la lactancia y crianza en el hogar durante los primeros años de vida.

Respecto a los distintos niveles socioeconómicos, los motivos de no asistencia son diferentes en función de los quintiles de ingreso. Entre quienes se encuentran en los quintiles superiores, aumenta la contratación de una persona cuidadora en el hogar como motivo de no asistencia al centro. Si bien se resisten a enviar a los niños a un centro infantil, la estrategia se basa en el cuidado domiciliario remunerado probablemente porque pueden pagar los costos que ello implica y por las experiencias previas de cuidado de calidad. Lo mismo ocurre con las mujeres que trabajan, entre las que es más frecuente la contratación de una persona remunerada como motivo de no asistencia a establecimientos de cuidado, respecto a las que no lo hacen $(4,0 \%$ vs $0,7 \%)$

\section{CUADRO 6}

MOTIVOS DE NO ASISTENCIA A CENTRO DE CUIDADO INFANTIL POR CONDICIÓN DE ACTIVIDAD DE LAS MUJERES MADRES Y QUINTILES DE INGRESO DE LOS HOGARES. TOTAL PAÍS, 2013

\begin{tabular}{|l|c|c|c|c|c|c|c|c|c|}
\hline & \multicolumn{3}{|c|}{ TRABAJO (\%) } & \multicolumn{5}{c|}{ QUINTILE (\%) } \\
\cline { 2 - 11 } & $\begin{array}{c}\text { NO } \\
\text { TRABAJA }\end{array}$ & TRABAJA & TOTAL & $\mathbf{1}$ & $\mathbf{2}$ - & 3. & $\mathbf{4}$ ( & 5. & TOTAL \\
\hline Edad temprana & 39,2 & 37,5 & 38,3 & 34,7 & 33 & 36,7 & 33,5 & 39,6 & 35,4 \\
\hline Cuidado familiar & 33,5 & 33,3 & 33,4 & 30,8 & 31,5 & 36,4 & 37,4 & 35,3 & 34,4 \\
\hline Problemas logísticos & 4,1 & 5,3 & 4,8 & 4,7 & 9,7 & 4 & 3,5 & 2,7 & 4,9 \\
\hline Precios excesivos & 2,8 & 2,1 & 2,4 & 3,5 & 4,1 & 2,5 & 1,9 & 1,1 & 2,6 \\
\hline $\begin{array}{l}\text { Calidad insuficiente/ } \\
\text { desconfianza }\end{array}$ & 2,5 & 2 & 2,2 & 2,5 & 3,2 & 2 & 1,7 & 2,7 & 2,4 \\
\hline $\begin{array}{l}\text { Cuidado contratado en } \\
\text { el hogar }\end{array}$ & 0,7 & 4 & 2,5 & 0,2 & 1,5 & 2,5 & 3,8 & 6,3 & 2,9 \\
\hline Miedo a enfermedades & 2 & 3,2 & 2,7 & 1,7 & 1,9 & 2,3 & 4,2 & 4,6 & 3 \\
\hline Otra o Ns/Nc & 15,2 & 12,5 & 13,6 & 20,9 & 14,9 & 13,6 & 14,1 & 7,7 & 14,5 \\
\hline Total & 100 & 100 & 100 & 100 & 100 & 100 & 100 & 100 & 100 \\
\hline
\end{tabular}

Nota: respuesta múltiple, los totales son la suma de respuestas de las madres en cada categoría y se excluyen los casos de personas responsables para los que se desconoce el quintil.

Fuente: Elaboración propia en base a ENDIS, INE-IECON-UCC.

Los problemas logísticos son un motivo de no concurrencia a un establecimiento de cuidado infantil que es más frecuente en lo manifestado por los sectores medios ( $2^{\circ}$ quintil). Son las mujeres que integran estos hogares las que se ven ante la tensión de tener que trabajar de forma remunerada, tener pocas probabilidades de pagar los altos costos que supone un centro infantil a jornada completa y al mismo tiempo no acceder a los servicios de cuidado público, enfocado fuertemente en el primer y segundo quintil.

En este sentido el 23,8\% de las madres encuentra dificultades para compatibilizar los horarios del centro de cuidado infantil con los de su trabajo remunerado, principalmente por la poca duración ofrecida 
por el centro infantil (18,2\%). Por tanto, es importante considerar esta demanda de extensión horaria en el caso de las mujeres que trabajan y que no pueden costear servicios de cuidado con extensión horaria, los cuales son más frecuentemente ofrecidas por el sector privado.

CUADRO 7

COMPATIBILIDAD DEL HORARIO DE CENTRO DE CUIDADO INFANTIL CON EL TRABAJO DE LAS MUJERES MADRES. TOTAL PAÍS, 2013

\begin{tabular}{|l|c|}
\hline & $\%$ \\
\hline Es compatible & 73,9 \\
\hline El horario es corto & 18,2 \\
\hline Abren muy tarde & $1,0^{*}$ \\
\hline Cierran muy temprano & 4,6 \\
\hline Ns/nc & $2,3^{*}$ \\
\hline Total & 100 \\
\hline
\end{tabular}

*Menos de 30 casos ponderados

Fuente: Elaboración propia en base a ENDIS, INE-IECON-UCC.

La ENDIS también indaga en los motivos de asistencia a los centros de cuidado infantil. El principal motivo de asistencia $(58,5 \%)$ mencionado es el beneficio que le reporta a los/as niños/as, y es más prevaleciente entre los quintiles inferiores que entre los superiores. El segundo gran motivo $(31,1 \%)$ es la necesidad de trabajar. Este motivo es más frecuente en los quintiles superiores respecto a los inferiores. De hecho éste es el primer motivo para la asistencia de los/as niños a un centro en el caso del quinto quintil. El 43,6\% del quintil cinco manifiesta como motivo principal el trabajo remunerado mientras que esto ocurre con tan solo el 9,8\% del primer quintil.

CUADRO 8

MOTIVOS DE ASISTENCIA AL CENTRO DE CUIDADO INFANTIL POR AÑOS DE ESTUDIO DE LOS/AS RESPONSABLES DE CRIANZA. TOTAL PAÍS, 2013

\begin{tabular}{|l|c|c|c|c|}
\hline \multirow{2}{*}{} & \multicolumn{4}{|c|}{ AÑOS DE ESTUDIO (\%) } \\
\cline { 2 - 5 } & \multirow{2}{*}{ O A 6 AÑOS } & $\begin{array}{c}\text { 7 A 12 } \\
\text { AÑOS }\end{array}$ & $\begin{array}{c}\text { 13 AÑOS } \\
\text { O MÁS }\end{array}$ & TOTAL \\
\hline Beneficios para los niños & 71,5 & 61,6 & 46,1 & 58,5 \\
\hline Trabajo remunerado & 13,8 & 29,3 & 43,4 & 31,1 \\
\hline $\begin{array}{l}\text { Recomendación de pediatra o } \\
\text { especialistas }\end{array}$ & 6,2 & 3,5 & 1,3 & 3,3 \\
\hline Estudio & 2,3 & 0,8 & 3,9 & 1,9 \\
\hline Tiempo Personal & 3,2 & 1,1 & 0 & 1,1 \\
\hline Otro o Ns/Nc & 3,1 & 3,7 & 5,4 & 4 \\
\hline Total & 100 & 100 & 100 & 100 \\
\hline
\end{tabular}

Fuente: Elaboración propia en base a ENDIS, INE-IECON-UCC. 


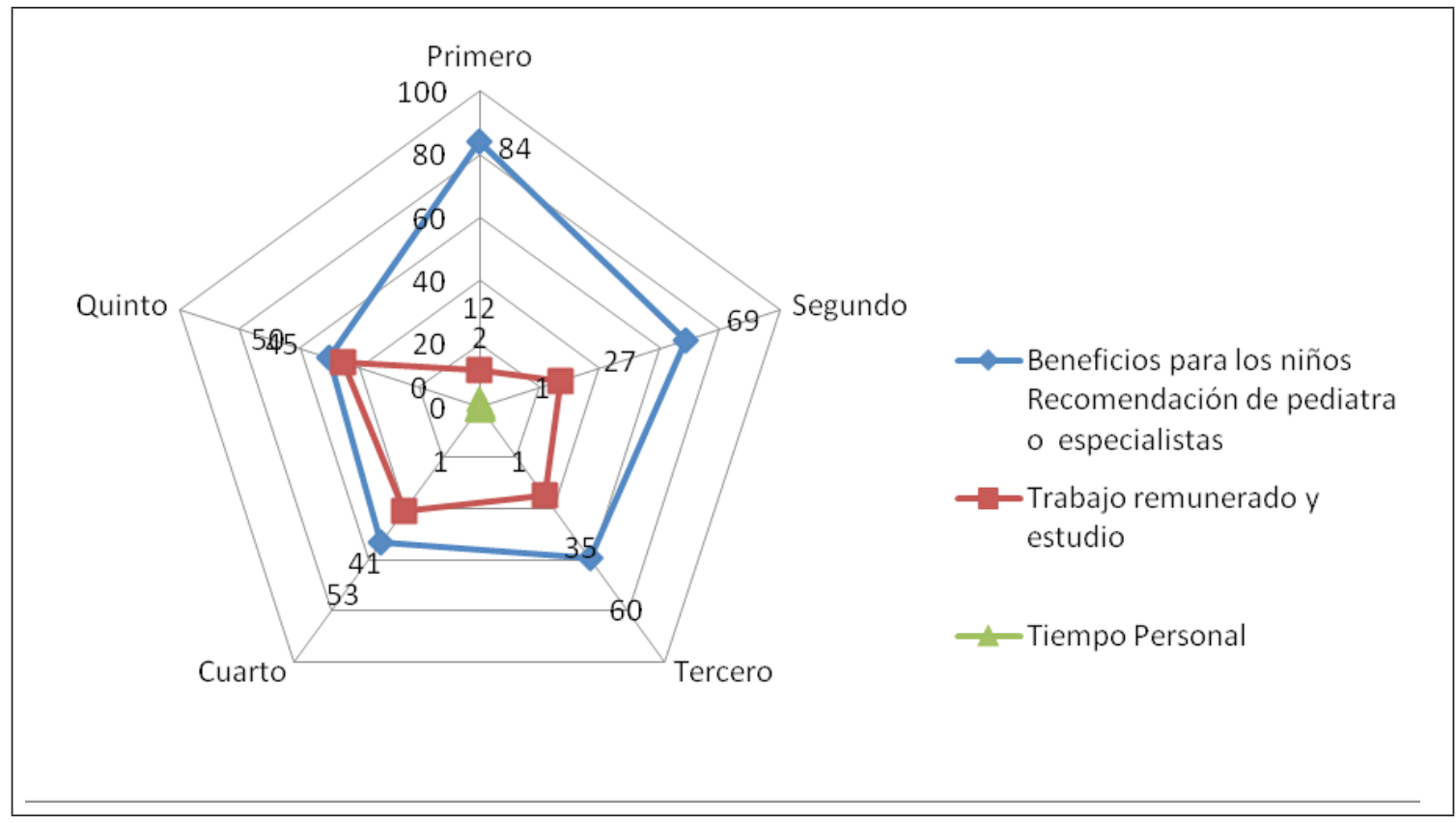

Fuente: Elaboración propia en base a ENDIS, INE-IECON-UCC.

El nivel educativo y el acceso al trabajo remunerado de las mujeres inciden en los motivos de asistencia al centro infantil, ya que las que poseen más años de educación (13 años o más) mencionan en mayor medida el motivo laboral que las que tienen hasta 6 años de estudio (43,4\% vs $13,8 \%$ y lo mismo ocurre con las mujeres que trabajan $(40,1 \%)$ respecto a las que no lo hacen $(9,1 \%)$.

En definitiva, la integración de los servicios de cuidado infantil en las estrategias de cuidado difieren según la situación socioeconómica del hogar y particularmente la situación laboral de las madres.

Entre aquellas mujeres que trabajan y pertenecen a niveles socioeconómicos más altos, existe la necesidad de cubrir la demanda de cuidados por medio de instituciones o la contratación de personas en el domicilio. Sin embargo, en el caso de las mujeres más pobres con poco acceso al mercado laboral y con bajos niveles educativos, la utilización del centro infantil responde a los beneficios para los/as niños/as y no se presenta como estrategia de compatibilización trabajo-cuidado.

Lo interesante a destacar es que en entre las mujeres que trabajan en forma remunerada y cuentan con nivel de educación universitaria, se observa una disonancia entre la estrategia deseable (cuidado familiar) y la realizada (cuidado institucional o contratado en domicilio). Este grupo de mujeres se enfrenta a las tensiones de articular el ámbito laboral con el doméstico, ambos con grandes exigencias en términos de tiempos, obligaciones y responsabilidades. 
Por otra parte, entre las mujeres pobres, con escaso acceso al trabajo remunerado, existe una concordancia entre la modalidad de cuidado infantil deseada mayoritariamente familiar y la efectivamente realizada. Probablemente la modalidad de cuidado deseada responde a una adaptación de las preferencias al campo de lo viable (ELSTER, 1988), es decir, ante la restricción de alternativas para el cuidado infantil (imposibilidad de contratación de cuidador/a remunerado en domicilio y de centros de cuidado a jornada completa) se opta por el cuidado familiar y se construye como la única opción deseada.

\section{EDAD EN QUE LOS/AS NIÑOS/AS COMIENZAN A ASISTIR A LOS CENTROS DE CUIDADO INFANTIL}

La ENDIS indaga la edad en que los/as niños/as comienzan a asistir a centros infantiles de cuidado. Dentro del grupo de niños/as que asiste a centros infantiles, 6 de cada 10 (59,6\%) lo hacen antes de los 12 meses.

La edad en que comienzan a asistir a los centros difiere significativamente entre los distintos quintiles de ingreso, ya que en los quintiles más bajos la entrada de los niños es más temprana. Mientras en el quintil uno el $71.8 \%$ los niños asiste antes del primer año, esto ocurre con el $48.5 \%$ del quinto quintil.

Esto se explica, en parte, por la modalidad de asistencia, particularmente la cantidad de horas que ellos asisten a los centros. En el caso de los quintiles más altos, cuando comienzan a asistir en edades más tardías, lo hacen en mayor número de días y mayor número de horas diarias, lo que seguramente está asociado a la necesidad de cubrir la jornada laboral completa y a la posibilidad de costear las extensiones horarias requeridas en los centros infantiles. Sin embargo, en los quintiles más bajos, si bien la asistencia es más temprana, lo hacen en menor número de días y horas diarias, no resultando por tanto una estrategia de compatibilización trabajo-cuidados. En el caso de los quintiles inferiores, la modalidad de asistencia está asociada a la oferta pública para estas edades, las cuales en la mayoría de los casos no superan las cuatro horas diarias. 


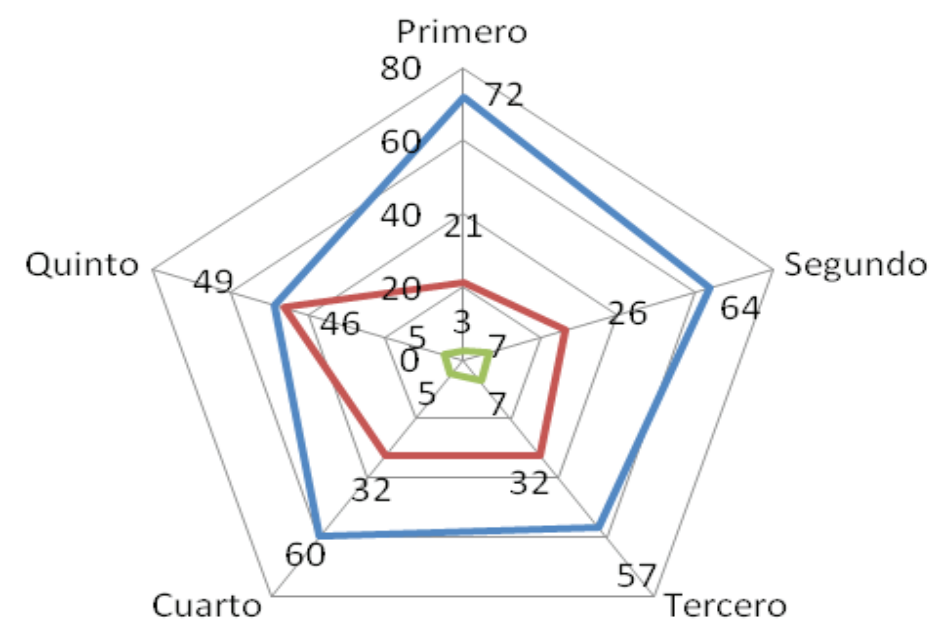

De 0 a 12 meses

De 13 a 24 meses

De 25 y más

Fuente: Elaboración propia en base a ENDIS, INE-IECON-UCC

Al observar la distribución de las edades de comienzo según el nivel educativo de la madre, se observa un fenómeno similar al presentado anteriormente por el nivel socioeconómico. Mientras que las mujeres de los niveles más bajos de educación envían a sus hijos a centros infantiles antes de los 12 meses en el 65,3\% de los casos, en las que tienen más años de educación, esto ocurre con el 54\% de los niños.

\section{CUADRO 9}

DISTRIBUCIÓN PORCENTUAL DE LA CANTIDAD DE DÍAS QUE ASISTEN Y HORAS SEMANALES PROMEDIO SEGÚN SITUACIÓN LABORAL DE LA MADRE. TOTAL PAÍS, 2013

\begin{tabular}{|l|c|c|c|c|c|c|}
\hline & \multicolumn{2}{|c|}{ NO TRABAJA } & \multicolumn{2}{c|}{ TRABAJA } & \multicolumn{2}{c|}{ TOTAL } \\
\cline { 2 - 7 } & PORCENTAJE & $\begin{array}{c}\text { HORAS } \\
\text { SEMANALES } \\
\text { PROMEDIO }\end{array}$ & PORCENTAJE & $\begin{array}{c}\text { HORAS } \\
\text { SEMANALES } \\
\text { PROMEDIO }\end{array}$ & PORCENTAJE & $\begin{array}{c}\text { HEMANALES } \\
\text { PROMEDIO }\end{array}$ \\
\hline Cinco días & 39,7 & 21 & 74,8 & 24 & 64,6 & 24 \\
\hline Cuatro días & 0,2 & 20 & 0,7 & 21 & 0,6 & 21 \\
\hline Tres días & 0,9 & 46 & 0,9 & 8 & 0,9 & 19 \\
\hline Dos días & 0,5 & 12 & 1,2 & 8 & 1 & 9 \\
\hline Un día & 58 & 4 & 22,1 & 2 & 32,6 & 3 \\
\hline $\begin{array}{l}\text { No sabe, No } \\
\text { contesta }\end{array}$ & 0,7 & 0 & 0,3 & 0 & 0,4 & 56 \\
\hline Total & 100 & 12 & 100 & 19 & 100 & 17 \\
\hline
\end{tabular}

Fuente: Elaboración propia en base a ENDIS, INE-IECON-UCC.

El porcentaje de niños/as que asiste cinco días a centros infantiles es significativamente mayor entre los que tienen madres que trabajan respecto a las que no $(74,8 \%$ y $39,7 \%$ respectivamente), detectándose una brecha de 3 horas semanales en el promedio de asistencia (24 y 21 
horas). De manera opuesta, el 58\% de los/as niños/as de las madres que no trabajan asiste un solo día a la semana a centros de cuidado infantil, 4 horas promedio semanales.

\section{ARTICULACIÓN TRABAJO-CUIDADOS}

Como se ha planteado al comienzo del artículo, uno de los elementos centrales de las estrategias de cuidado es la articulación entre el trabajo remunerado y el trabajo de cuidados, debido a las tensiones y los costos que genera específicamente para las mujeres, que como se ha mostrado, son las principales cuidadoras infantiles.

En primer lugar, uno de los datos que proporciona la encuesta es el acceso de las mujeres trabajadoras a las licencias maternales y la reducción de horario para cuidados o licencias parentales. El 65,4\% de las madres encuestadas tiene acceso a licencias por maternidad en su trabajo y el 60,7\% tiene derecho a reducción de horario para cuidados. Sin embargo, esto difiere entre las personas de distintos niveles socioeconómicos. Dentro de las mujeres no pobres que trabajan el 71,9\% tiene derecho a las licencias maternales y tan solo el 33,5\% de las pobres lo tiene, presentándose una situación similar con el derecho a la reducción horaria para cuidados.

\section{CUADRO 10}

DERECHO A LICENCIA POR MATERNIDAD DE LAS MUJERES QUE TRABAJAN POR POBREZA DEL HOGAR. TOTAL PAÍS, 2013

\begin{tabular}{|l|c|c|c|}
\hline & NO POBRE (\%) & POBRE (\%) & TOTAL (\%) \\
\hline $\begin{array}{l}\text { Tiene derecho a licencia } \\
\text { por maternidad }\end{array}$ & 71,9 & 33,5 & 65,4 \\
\hline $\begin{array}{l}\text { No tiene derecho a licencia } \\
\text { por maternidad }\end{array}$ & 27,2 & 62,7 & 33,1 \\
\hline Ns Nc & $1,0^{*}$ & $3,8^{*}$ & $1,4^{*}$ \\
\hline Total & 100 & 100 & 100 \\
\hline
\end{tabular}

*Menos de 30 casos ponderados

Fuente: Elaboración propia en base a ENDIS, INE-IECON-UCC.

El dato de que prácticamente 6 de cada 10 mujeres pobres no accedan a las licencias maternales es alarmante y seguramente está relacionado a los tipos de trabajo a los que acceden, muchos de ellos sin cobertura de la seguridad social (por ejemplo el 56,5\% de las trabajadoras domésticas no aportaban a la seguridad social en Uruguay en $2013^{6}$ ) o por cuenta propia como monotributistas. Las modificaciones en las licencias por maternidad, Ley $N^{\circ}$ 19.161, realizadas en el año 2013, implican que las mujeres que están en situación de monotributistas (las mismas representaban en 2013 más de la mitad de los/as monotributistas³) 
tengan derecho a licencias por maternidad. Por tanto, cabría esperar cambios en estos indicadores en los próximos años.

En relación con estos datos, la posibilidad de acceder a una licencia por maternidad se incrementa con el nivel educativo de dichas mujeres. Entre aquellas mujeres con 13 o más años de educación, el $82.9 \%$ tiene derecho a la licencia maternal mientras que esto ocurre con el $41.6 \%$ de las que cuentan con hasta seis años de estudio. Es probable que el mayor acceso a licencias de las mujeres más educadas esté relacionado a la mejor calidad de los empleos, pero sobre todo a la mayor cobertura de seguridad social con que cuentan.

CUADRO 11

ACCESO A LICENCIA POR MATERNIDAD DE LAS MUJERES QUE TRABAJAN POR AÑOS DE ESTUDIO. TOTAL PAÍS, 2013

\begin{tabular}{|l|c|c|c|c|}
\hline & O A 6 AÑOS & 7 A 12 AÑOS & 13 AÑOS O MÁs & TOTAL \\
\hline $\begin{array}{l}\text { Tiene derecho a licencia } \\
\text { por maternidad }\end{array}$ & 41,6 & 60,1 & 82,9 & 65,5 \\
\hline $\begin{array}{l}\text { No tiene derecho a licencia } \\
\text { por maternidad }\end{array}$ & 57,3 & 37,7 & 16,5 & 33,0 \\
\hline Ns/nc & 1,1 & 2,1 & 0,6 & 1,5 \\
\hline Total & 100 & 100 & 100 & 100 \\
\hline
\end{tabular}

Fuente: Elaboración propia en base a ENDIS, INE-IECON-UCC.

La encuesta también indaga sobre la interrupción del trabajo remunerado por nacimiento de un/una hijo/a, consultando sobre la interrupción de más de tres meses del trabajo, es decir, más tiempo del que provee la licencia maternal (13 o 14 semanas). La mitad de las mujeres $(50,6 \%)$ interrumpió el trabajo, mientras que la otra mitad no lo hizo.

\section{CUADRO 12}

MUJERES QUE INTERRUMPIERON SU TRABAJO POR NACIMIENTO DE UN/A HIJO/A, POR NIVEL EDUCATIVO Y POBREZA EN EL HOGAR. TOTAL PAÍS, 2013

\begin{tabular}{|l|c|c|c|c|c|c|c|}
\hline & \multicolumn{3}{|c|}{ POBREZA (\%) } & \multicolumn{4}{c|}{ AÑOS DE ESTUDIO (\%) } \\
\cline { 2 - 8 } & NO POBRE & POBRE & TOTAL & O A 6 & 7 A 12 & 13 EN ADELANTE & TOTAL \\
\hline Interrumpió su trabajo & 50,8 & 49,3 & 50,5 & 50,7 & 50,4 & 51 & 50,6 \\
\hline No interrumpió & 49 & 50,4 & 49,3 & 48,9 & 49,4 & 48,9 & 49,2 \\
\hline Ns/Nc & 0,2 & 0,3 & 0,2 & 0,4 & 0,2 & 0,1 & 0,2 \\
\hline Total & 100 & 100 & 100 & 100 & 100 & 100 & 100 \\
\hline
\end{tabular}

Fuente: Elaboración propia en base a ENDIS, INE-IECON-UCC.

Este dato no varía en función de la pobreza del hogar ni en los distintos niveles educativos, lo que da cuenta nuevamente de la división sexual del trabajo que favorece el abandono temporal o definitivo del trabajo remunerado de las mujeres para dedicarse al cuidado y de la rigidez del mercado laboral, que no permite otras formas de articulación 
trabajo-familia que le permitan a los hogares cuidar al mismo tiempo que eviten que las mujeres abandonen sus empleos.

Cuando se indaga en el tiempo que las mujeres estuvieron sin trabajar luego del nacimiento de sus hijos, el 68\% lo hizo hasta un año, aunque no es despreciable que el 24,4\% estuvo entre 1 y 3 años sin trabajar. La necesidad de cuidado infantil bajo un modelo familista de cuidados que asigna el rol de cuidados a las mujeres y que se caracteriza por la gran ausencia de servicios estatales de calidad a edades tempranas, tiene como consecuencia que las mujeres asumen individualmente los costos.

\section{CUADRO 13}

TIEMPO QUE LAS MUJERES ESTUVIERON SIN TRABAJAR LUEGO DEL NACIMIENTO DE SUS HIJOS. TOTAL PAÍS, 2013

\begin{tabular}{|l|c|}
\hline & $\%$ \\
\hline Seis meses & 35,3 \\
\hline Entre seis meses y un año & 32,7 \\
\hline Entre uno y dos años & 17,1 \\
\hline Entre dos y tres años & 7,3 \\
\hline Entre tres y cuatro años & 2,5 \\
\hline Más de cuatro años & 5,1 \\
\hline Total & 100 \\
\hline
\end{tabular}

Fuente: Elaboración propia en base a ENDIS, INE-IECON-UCC.

Por su parte, el tiempo de interrupción en el mercado de trabajo se incrementa en el caso de las mujeres pobres. Estas últimas pasan períodos más largos de tiempo fuera del mercado. Lo mismo ocurre con el nivel educativo, ya que a menor nivel educativo es mayor el tiempo que transcurre fuera del mercado laboral. El hecho de que las mujeres pasen grandes períodos de tiempo fuera del mercado laboral condiciona su reinserción futura y su trayectoria, siendo el comienzo de la vida en pareja, el embarazo y los cuidados de niños/as hitos que interrumpen la trayectoria laboral de las mujeres (URUGUAY, 2012). La lógica de funcionamiento del mercado laboral está basada en la acumulación de saberes y experiencias y en trayectorias continuas, tal como ocurre más frecuentemente con los varones. Las trayectorias discontinuas más características de las mujeres condicionan sus carreras en comparación con los varones y dificulta su reinserción, lo que las vuelve nuevamente responsables exclusivas de los costos del cuidado. ${ }^{8}$ Esto tiene consecuencias no solo en su autonomía económica durante las etapas iniciales de la familia cuando los hijos/as son pequeños/as sino en el futuro, a la hora del retiro del mercado de trabajo. Durante la vejez, las mujeres se ven perjudicadas por sus trayectorias discontinuas, con menor acceso a las jubilaciones y con montos inferiores, lo que nuevamente las sitúa en condiciones desventajosas desde el punto de vista de la autonomía económica. 
CUADRO 14

TIEMPO QUE LAS MUJERES ESTUVIERON SIN TRABAJAR LUEGO DEL NACIMIENTO DE SUS HIJOS POR POBREZA EN EL HOGAR Y NIVEL EDUCATIVO (CANTIDAD DE AÑOS DE ESTUDIO). TOTAL PAÍS, 2013

\begin{tabular}{|l|c|c|c|c|c|c|c|}
\hline \multirow{2}{*}{} & \multicolumn{3}{|c|}{ POBREZA (\%) } & \multicolumn{4}{c|}{ NIVEL EDUCATIVO (\%) } \\
\cline { 2 - 10 } & $\begin{array}{c}\text { NO } \\
\text { POBRES }\end{array}$ & POBRES & TOTAL & 0 A 6 & 7 A 12 & 13 Y MÁs & TOTAL \\
\hline Hasta un año & 70,6 & 59,4 & 68,1 & 58,3 & 64,2 & 84,1 & 68 \\
\hline Entre un año y dos & 16,5 & 21,6 & 17,7 & 21,7 & 19 & 9,1 & 17 \\
\hline Entre dos años y tres & 6,3 & 8,9 & 6,9 & $12,8^{*}$ & 7 & $4,0^{*}$ & 7,3 \\
\hline Más de tres años & 6,5 & 10,1 & 7,3 & $7,1^{*}$ & 9,9 & $2,8^{*}$ & 7,6 \\
\hline Total & 100 & 100 & 100 & 100 & 100 & 100 & 100 \\
\hline
\end{tabular}

*Menos de 30 casos ponderados

Fuente: Elaboración propia en base a ENDIS, INE-IECON-UCC.

Los datos referidos a la práctica de interrumpir el trabajo remunerado en el caso de las mujeres ante la llegada de los hijos/as son coincidentes con lo encontrado en el nivel de los discursos. El mandato de género que rige para el cuidado de los niños pequeños plantea de manera determinante la mayor disposición de las mujeres a la interrupción o abandono del empleo ante las necesidades de cuidado. Más de un tercio de las mujeres manifiesta que estarían dispuestas a abandonar su empleo en caso de tener que cuidar a un/a hijo/a menor de 1 año. El 21,5\% de ellas abandonaría provisoriamente su empleo y $12,6 \%$ lo haría en forma definitiva. En el caso de los varones, estos porcentajes se reducen al 10,1 y 5,1\%, respectivamente (BATTHYÁNY; GENTA; PERROTTA, 2013).

\section{ESTEREOTIPOS DE GÉNERO EN LA CRIANZA}

La ENDIS indaga sobre algunos estereotipos de género presentes en la forma en que se distribuye el cuidado entre varones y mujeres. En el siguiente apartado se analizan las opiniones de la población sobre las obligaciones respecto al cuidado infantil que tienen madres y padres. La ENDIS releva dichas obligaciones a través del acuerdo o desacuerdo con frases sobre la socialización de hijos e hijas y referidas a los roles de varones y mujeres en el trabajo remunerado y en los cuidados.

Alrededor del 10\% de los/as encuestados/as está de acuerdo con frases que vinculan a las mujeres a las tareas del hogar: "A las niñas hay que enseñarles que el lugar de la mujer es en la casa" y "A los varones hay que enseñarles a cuidarse por sí mismos y a las niñas las tiene que cuidar uno" y a los varones a asumir un rol de protección y de autoridad: "A los varones hay que educarlos para que sepan mandar en su casa".

El acuerdo con dichos roles diferenciados es notoriamente más alto entre la población pobre, llegando a representar cerca del 18\% de este grupo (mayor al 10\% de la población general). En la población no 
pobre el nivel de acuerdo con las tres frases es menor al promedio y refleja alrededor del 6\% de esta población. La frase "A las niñas hay que enseñarles que el lugar de la mujer es en la casa" es la que presenta mayor nivel de acuerdo, 19,5\% de la población pobre y 6,6\% de los no pobres.

Así, la división sexual del trabajo y los contratos de género son más rígidos y tradicionales entre las personas de menores niveles socioeconómicos, lo cual coincide con los datos sobre representaciones sociales del cuidado, en los cuales se encuentra mayor familismo entre las personas de niveles socioeconómicos bajos, en relación a los medioaltos (BATTHYÁNY; GENTA; PERROTA, 2013).

La situación laboral también incide en el nivel de acuerdo con dichas frases. Las madres que no trabajan de forma remunerada tienden a estar más de acuerdo con las frases que se refieren a estereotipos más tradicionales, alrededor del 14\%, mientras que entre las que trabajan, menos del 6\% está de acuerdo.

\section{CUADRO 15}

ACUERDO DE LAS MUJERES MADRES DE LOS NIÑOS/AS CON LAS SIGUIENTES AFIRMACIONES POR ACTIVIDAD LABORAL Y NIVEL EDUCATIVO (AÑOS DE ESTUDIO) DE LAS MISMAS. TOTAL PAÍS, 2013

\begin{tabular}{|l|c|c|c|c|c|c|c|}
\hline \multirow{2}{*}{} & \multicolumn{3}{|c|}{ TRABAJO (\%) } & \multicolumn{3}{c|}{ AÑOS DE ESTUDIO (\%) } \\
\cline { 2 - 7 } & $\begin{array}{c}\text { NO } \\
\text { TRABAJA }\end{array}$ & TRABAJA & TOTAL & O A 6 & 7 A 12 & 13 Y MÁS & TOTAL \\
\hline $\begin{array}{l}\text { A los varones hay que } \\
\text { educarlos para que sepan } \\
\text { mandar en su casa }\end{array}$ & 14,5 & 6,2 & 9,4 & 26,1 & 7,2 & 0,5 & 9,4 \\
\hline $\begin{array}{l}\text { A los varones hay que } \\
\text { enseñarles a cuidarse por sí } \\
\text { mismos y a las niñas las tiene } \\
\text { que cuidar uno }\end{array}$ & 13,3 & 6,6 & 9,2 & 25,2 & 6,7 & 1,4 & 9,2 \\
\hline $\begin{array}{l}\text { A las niñas hay que } \\
\text { enseñarles que el lugar de la } \\
\text { mujer es en la casa }\end{array}$ & 15,7 & 7,8 & 10,9 & 30,6 & 7,8 & 1,2 & 10,8 \\
\hline
\end{tabular}

Fuente: Elaboración propia en base a ENDIS, INE-IECON-UCC.

El nivel educativo también diferencia claramente entre quienes acuerdan con las frases mencionadas. Entre aquellos/as que cuentan con menos años de educación, el nivel de acuerdo con las frases aumenta considerablemente. La frase “A las niñas hay que enseñarles que el lugar de la mujer es en la casa" llega ser del 30,6\% de la población con nivel primario de la educación. En el caso de la población con más años de educación estos porcentajes bajan, siendo de alrededor del 7\% entre los que tienen un nivel de educación secundaria o similar y de menos del $2 \%$ entre quienes poseen educación terciaria.

Este deber ser del cuidado impacta sobre la dedicación de tiempo a esta tarea por parte de unos y otras. Evidencia de este hecho puede observarse en la tasa de actividad según cantidad de hijos, ya que mientras los varones incrementan su dedicación al trabajo remunerado en el mercado cuando 
tienen hijos/as, las mujeres reducen su participación dedicándose en mayor medida al cuidado directo (URUGUAY, 2013).

En definitiva, los contratos de género vigentes en Uruguay asignan a las mujeres la responsabilidad del cuidado familiar de niños y niñas. El tiempo destinado al cuidado de hijas e hijos y al trabajo doméstico está fuertemente moralizado: está presente la idea de lo bueno y el deber, y se observa una mayor carga de responsabilidad en la ejecución de las tareas de cuidado a las mujeres.

\section{REFLEXIONES FINALES}

En el siguiente apartado se retoman las principales ideas del artículo. Las estrategias de cuidado infantil se encuentran basadas fundamentalmente en la red familiar y de las mujeres, tanto sean madres, abuelas y hermanas. Es decir que el familismo que se presenta en el discurso del cuidado infantil ideal encuentra un correlato en las prácticas, es decir, en cómo los integrantes de los hogares solucionan el cuidado infantil.

La asistencia de los niños a centros de cuidado infantil, si bien podría contribuir en parte o totalmente al cuidado de calidad de los niños pequeños durante la jornada laboral, es utilizada tan solo en 1 de cada 3 niños. Las razones de dicha inasistencia están relacionadas muy fuertemente con las representaciones sociales del cuidado, las que se manifiestan en la idea de que son edades muy tempranas para enviar a los hijos al centro infantil. Esta idea proviene sobre todo de un predominante discurso médico, que enfatiza que el cuidado ideal es el que se realiza en el hogar a cargo de familiares. Por su parte, las situaciones deseables para el cuidado infantil reafirman este rol de las familias como principales proveedoras del mismo. La mayor parte de la población considera que la situación deseable durante la jornada laboral es el cuidado infantil provisto por miembros de la pareja o familiares cercanos.

En segundo lugar, las estrategias de cuidado, al igual que las representaciones sociales del cuidado, varían en los distintos niveles socioeconómicos. Cuando observamos las estrategias de los hogares por niveles socioeconómicos, se evidencia que los hogares no pobres integran la contratación de una persona remunerada como parte de su estrategia de cuidados, mientras que esto no ocurre en los pobres. La asistencia a centros de cuidado infantil es más alta entre quienes no son pobres y entre las madres que trabajan respecto a las que no lo hacen.

La tendencia a la integración de actores extrafamiliares depende de las posibilidades económicas de costearlo, dado que se suma al eje de las desigualdades de género una desigualdad de clase. Las representaciones sociales sobre las situaciones de cuidado infantil más deseables también son distintas por nivel socioeconómico. Los sectores 
de niveles más altos son menos familistas que los de niveles más bajos. Esto último abona a la hipótesis de que parte del discurso familista puede ser una preferencia adaptativa por no haber experimentado la posibilidad de contar con centros de cuidados o personas externas al hogar (del Estado o el mercado) para los cuidados de niños/as.

En tercer lugar, los resultados muestran la permanencia de estereotipos de género basados en roles rígidos producto de la división sexual del trabajo tradicional entre mujeres y varones. Asimismo, estos roles son apropiados mayormente por los quintiles más bajos, por las personas de los niveles educativos más bajos y por las mujeres que no trabajan.

Respecto a la articulación trabajo-cuidados, el derecho a las licencias por maternidad difiere entre los quintiles de ingreso de las mujeres producto de los tipos de trabajo a los que acceden, generando una desigualdad en la articulación trabajo-cuidados entre las distintas mujeres. Por otro lado, es notorio el tiempo de interrupción del mercado laboral que experimentan las mujeres ante la llegada de los hijos. Ante la inexistencia de políticas públicas de cuidado infantil, son las mujeres las que, cargando con el deber ser asociado al cuidado directo de los niños pequeños, abandonan momentánea o definitivamente el empleo ante la necesidad de cuidado. Los resultados evidencian que esto sucede de forma independiente del quintil de ingresos y el nivel educativo, aunque los años que se pasa fuera del mercado se incrementan en el caso de las mujeres pobres, para quienes probablemente les cuesta en mayor medida acceder a un empleo que les permita articular cuidados con trabajo remunerado. El abandono tiene consecuencias en términos de la autonomía económica presente y futura de las mujeres, quienes de esta forma asumen individualmente los costos del cuidado.

\section{BIBLIOGRAFÍA}

BATTHYÁNY, Karina. Los tiempos del Cuidado en Uruguay. In: BATTHYÁNY, Karina (Coord.). Los tiempos del bienestar social. Género, trabajo no remunerado y cuidados en Uruguay. Montevideo: Doble Clic, 2015.

BATTHYÁNY, Karina; GENTA, Natalia; PERROTTA, Valentina. La población uruguaya y el cuidado. Análisis de representaciones sociales y propuestas para un Sistema de Cuidados en Uruguay. Montevideo: Ministerio de Desarrollo Social, 2013.

BATTHYÁNY, Karina; GENTA, Natalia; PERROTTA, Valentina. Los cuidados no remunerados en salud: el rol de las familias y las mujeres. Primeros resultados de la Encuesta Nacional de Cuidados no Remunerados en Salud. En El Uruguay desde la Sociología 12. DS-FCS-UDELAR. Montevideo, 2014.

BATTHYÁNY, Karina; GENTA, Natalia; PERROTTA, Valentina. Uso del tiempo y desigualdades de género en el trabajo no remunerado. In: BATTHYÁNY, Karina (Coord.) Los tiempos del bienestar social. Género, trabajo no remunerado y cuidados en Uruguay. Montevideo: Doble Clic, 2015.

BECK, Ulrich; BECK-GERNSHEIM, Elisabeth. Amor a distancia: nuevas formas de vida en la era global. Buenos Aires: Paidós Contextos, 2012. 
BLOFIELD, Merike; MARTINEZ, Juliana. Trabajo, familia y cambios en la política pública en América Latina: equidad, maternalismo y corresponsabilidad. Revista CEPAL, n. 114, p. 117-125, 2014.

BOCK, Gisela; DUDEN, Barbara. Trabajo por amor, amor como trabajo. Desarrollo, Revista de la Sociedad Internacional para el desarrollo, Madrid, n. 2, p. 39-50, 1985.

CARRASCO, Cristina; BORDERÍAS, Cristina; TORNS, Teresa. Introducción. El trabajo de cuidados: antecedentes históricos y debates actuales. En: CARRASCO, Cristina; BORDERÍAS, Cristina; TORNS, Teresa (Ed.). El trabajo de cuidados: historia, teorías y política. Madrid: Catarata, 2011.

ELSTER, Jon. Uvas amargas. Sobre la subversión de la racionalidad. Barcelona: Edicions 62, 1988.

FERNÁNDEZ, Juan Antonio; TOBÍO, Constanza. Conciliar las responsabilidades familiares y laborales: políticas y prácticas sociales. OIT, 2006. (Documento de Trabajo 79/2005).

NARANJA SOTELO, Martín. Monotributo: descripción y análisis de su evolución. Asesoría en Políticas de Seguridad Social Asesoría General en Seguridad Social. Banco de Previsiòn Social. Montevideo, 2014.

TOBÍO, Constanza. Conciliación o contradicción: cómo hacen las madres trabajadoras. Revista Española de Investigaciones Sociológicas, v. 97, n. 2, p. 155-186, 2002.

TRONTO, Joan. Creating caring institutions: politics, plurality, and purpose. Ethics and Social Welfare, v. 4, n. 2, p. 158-171, 2010.

URUGUAY. Ministerio de Desarrollo Social. Cuidados como sistema. Propuesta para un modelo solidario y corresponsable de cuidados en Uruguay. Montevideo: MIDES, 2014.

URUGUAY. Ministerio de Desarrollo Social. Contribuciones para comprender y medir la pobreza desde la perspectiva de género. Montevideo: MIDES, 2012. (Cuadernos del Sistema de Información de Género, n. 4).

URUGUAY. Ministerio de Desarrollo Social. Estadísticas de género: evolución de los indicadores de género en el período 2009-2013 Montevideo: UNFPA, MIDES, INMUJERES, 2013.

WALLACE, Claire. Household strategies: their conceptual relevance and analytical scope in social research. Sociology, v. 36, n. 2 p. 275-292, 2002.

\section{KARINA BATTHYÁNY}

Universidad de la República, Uruguay

karina.batthyany@cienciassociales.edu.uy

\section{NATALIA GENTA}

Universidad de la República, Uruguay

natalia.genta@gmail.com

\section{SOL SCAVINO}

Universidad de la República, Uruguay

solscavino@gmail.com 